\title{
Nonlocal cauchy problem for delay fractional integrodifferential equations of neutral type
}

Fang Li

Correspondence: fangli860@gmail. com

School of Mathematics, Yunnan Normal University, Kunming 650092, People's Republic of China

\section{Abstract}

This article concerns the existence of mild solutions for the fractional integrodifferential equations of neutral type with finite delay and nonlocal conditions in a Banach space $X$. The existence of mild solutions is proved by means of measure of noncompactness. As an application, the existence of mild solutions for some integrodifferential equation is obtained.

Mathematics Subject Classification: 34K05; 34A12.

Keywords: fractional integrodifferential equation, mild solution, finite delay, nonlocal condition, neutral type, measure of noncompactness

\section{Introduction}

The fractional differential equations have received increasing attention during recent years and have been studied extensively (see, e.g., [1-10] and references therein) since they can be used to describe many phenomena arising in viscoelasticity, electrochemistry, control, porous media, electromagnetic, etc.

Moreover, the Cauchy problem for various delay equations in Banach spaces has been receiving more and more attention during the past decades (see, e.g., $[2,3,6,7,10-12])$.

As in [5,8,13-15] and the related references given there, we pay attention to the nonlocal condition because in many cases a nonlocal condition $v(0)+g(v)=v_{0}$ is more realistic than the classical condition $v(0)=v_{0}$ in treating physical problems. To the author's knowledge, few articles can be found in the literature for the solvability of the fractional order delay integrodifferential equations of neutral type with nonlocal conditions.

In this article, we concern with the following nonlocal neutral delay fractional integrodifferential equations

$$
\begin{aligned}
{ }^{c} D^{q}\left(v(t)-h\left(t, v_{t}\right)\right) & =A v(t)+f\left(t, v_{t}\right)+\int_{0}^{t} a\left(t, s, v_{s}\right) d s, \quad t \in[0, T], \\
v(t) & =g(v)(t)+\phi(t), \quad t \in[-r, 0],
\end{aligned}
$$

where $T>0,0<q<1,0<r<\infty$. The fractional derivative is understood here in the Caputo sense. $X$ is a separable Banach space. $A$ is a closed operator. Here $h:[0, T] \times C$ $([-r, 0], X) \rightarrow X, f:[0, T] \times C([-r, 0], X) \rightarrow X, a: \Delta \times C([-r, 0], X) \rightarrow X(\Delta=\{(t, s) \in[0$,

(C) 2012 Li; licensee Springer. This is an Open Access article distributed under the terms of the Creative Commons Attribution License (http://creativecommons.org/licenses/by/2.0), which permits unrestricted use, distribution, and reproduction in any medium, provided the original work is properly cited. 
$T] \times[0, T]: t \geq s\}), g: C([-r, 0], X) \rightarrow C([-r, 0], X), \varphi \in C([-r, 0], X)$, where $C([a, b], X)$ denotes the space of all continuous functions from $[a, b]$ to $X$.

For any continuous function $v$ defined on $[-r, T]$ and any $t \in[0, T]$, we denote by $v_{t}$ the element of $C([-r, 0], X)$ defined by $v_{t}(\theta)=v(t+\theta), \theta \in[-r, 0]$.

This article is organized as follows. In Section 2, we recall some basic definitions and preliminary results. In Section 3, we give the existence theorem of mild solution of (1.1) and its proof. In the last section, an example is given to show an application of the abstract result.

\section{Preliminaries}

Throughout this article, we denote by $X$ a separable Banach space with norm $\|\cdot\|$, by $L(X)$ the Banach space of all linear and bounded operators on $X$, and by $C([a, b], X)$ the space of all $X$-valued continuous functions on $[a, b]$ with the supremum norm as follows:

$$
\|x\|_{[a, b]}=\|x\|_{C([a, b], X)}=\sup \{\|x(t)\|: t \in[a, b]\}, \text { for any } x \in C([a, b], X) .
$$

Moreover, we abbreviate $\|u\|_{L^{p}\left([0, T], \mathbf{R}^{+}\right)}$with $\|u\|_{L^{p}}$, for any $u \in L^{p}\left([0, T], \mathbf{R}^{+}\right)$.

In this article, $A$ is the infinitesimal generator of an uniformly bounded analytic semigroup of linear operators $\left\{S(t)_{\mathrm{t} \geq 0}\right.$ in $X$. We will assume that $0 \in \rho(A)(\rho(A)$ is the resolvent set of $X$ ) and that

$$
\|S(t)\| \leq M, \text { for all } t \in[0, T] .
$$

Under these conditions it is possible to define the fractional power $(-A)^{\alpha}, 0<\alpha<1$, as closed linear operator on its domain $D(-A)$. Recall the knowledge in [16], we have

(1) there exists a constant $M_{0}>0$ such that

$$
\left\|(-A)^{-\alpha}\right\| \leq M_{0} .
$$

(2) for any $\alpha \in(0,1)$, there exists a positive constant $C_{\alpha}$ such that

$$
\left\|(-A)^{\alpha} S(t)\right\| \leq \frac{C_{\alpha}}{t^{\alpha}} .
$$

Let us recall the following known definitions. For more details see [9].

Definition 2.1. [9] The fractional integral of order $q$ with the lower limit zero for a function $f \in A C[0, \infty)$ is defined as

$$
I^{q} f(t)=\frac{1}{\Gamma(q)} \int_{0}^{t}(t-s)^{q-1} f(s) d s, \quad t>0, \quad 0<q<1,
$$

provided the right side is point-wise defined on $[0, \infty)$, where $\Gamma(\cdot)$ is the gamma function.

Definition 2.2. [9] Riemann-Liouville derivative of order $q$ with the lower limit zero for a function $f \in A C[0, \infty)$ can be written as

$$
{ }^{L} D^{q} f(t)=\frac{1}{\Gamma(1-q)} \frac{d}{d t} \int_{0}^{t}(t-s)^{-q} f(s) d s, \quad t>0, \quad 0<q<1 .
$$


Definition 2.3. [9] The Caputo derivative of order $q$ for a function $f \in A C[0, \infty)$ can be written as

$$
{ }^{c} D^{q} f(t)={ }^{L} D^{q}(f(t)-f(0)), \quad t>0, \quad 0<q<1 .
$$

Remark 2.4. (1) If $f(t) \in C^{1}[0, \infty)$, then

$$
{ }^{c} D^{q} f(t)=\frac{1}{\Gamma(1-q)} \int_{0}^{t}(t-s)^{-q} f^{\prime}(s) d s=I^{1-q} f^{\prime}(t), \quad t>0, \quad 0<q<1 .
$$

(2) The Caputo derivative of a constant is equal to zero.

We will need the following facts from the theory of measures of noncompactness and condensing maps (see, e.g., $[17,18]$ ).

Definition 2.5. Let $E$ be a Banach space and $(\mathcal{A}, \geq)$ a partially ordered set. A function $\beta: P(E) \rightarrow \mathcal{A}$ is called a measure of noncompactness (MNC) in $E$ if

$$
\beta(\overline{\mathrm{CO}}(\Omega))=\beta(\Omega) \quad \text { for every } \Omega \in P(E),
$$

where $P(E)$ denotes the class of all nonempty subsets of $E$.

A MNC $\beta$ is called:

(i) monotone, if $\Omega_{0}, \Omega_{1} \in P(E), \Omega_{0} \subset \Omega_{1}$ implies $\beta\left(\Omega_{0}\right) \leq \beta\left(\Omega_{1}\right)$;

(ii) nonsingular, if $\beta\left(\left\{a_{0}\right\} \cup \Omega\right)=\beta(\Omega)$ for every $a_{0} L E, \Omega \in P(E)$;

(iii) invariant with respect to union with compact sets, if $\beta(\{D\} \cup \Omega)=\beta(\Omega)$ for every relatively compact set $D \subset E, \Omega \in P(E)$.

If $\mathcal{A}$ is a cone in a normed space, we say that the MNC $\beta$ is

(iv) algebraically semiadditive, if $\beta\left(\Omega_{0}+\Omega_{1}\right) \leq \beta\left(\Omega_{0}\right)+\beta\left(\Omega_{1}\right)$ for each $\Omega_{0}, \Omega_{1} L P$

$(E)$;

(v) regular, if $\beta(\Omega)=0$ is equivalent to the relative compactness of $\Omega$;

(vi) real, if $\mathcal{A}$ is $[0,+\infty)$ with the natural order.

As an example of the MNC possessing all these properties, we may consider the Hausdorff MNC

$$
\mathcal{X}(\Omega)=\inf \{\varepsilon>0: \Omega \text { has a finite } \varepsilon-\text { net }\} .
$$

Now, let $G:[0, \tilde{h}] \rightarrow P(E)$ be a multifunction. It is called:

(i) integrable, if it admits a Bochner integrable selection $\mathfrak{g}:[0, \tilde{h}] \rightarrow E, \quad \mathfrak{g}(t) \in G(t)$ for a.e. $t \in[0, \tilde{h}]$

(ii) integrably bounded, if there exists a function $\vartheta \in L^{1}([0, \tilde{h}], E)$ such that

$$
\|G(t)\|:=\sup \{\|\mathfrak{g}\|: \mathfrak{g} \in G(t)\} \leq \vartheta(t) \text { a.e } t \in[0, \tilde{h}]
$$

We present the following assertion about $\chi$-estimates for a multivalued integral [[18], Theorem 4.2.3].

Proposition 2.6. For an integrable, integrably bounded multifunction $G:[0, \tilde{h}] \rightarrow P(X)$ where $X$ is a separable Banach space, let 


$$
\chi(G(t)) \leq \mathfrak{q}(\mathrm{t}), \quad \text { for a.e. } t \in[0, \tilde{h}]
$$

where $\mathfrak{q} \in L_{+}^{1}([0, \tilde{h}])$. Then $\chi\left(\int_{0}^{t} G(s) d s\right) \leq \int_{0}^{t} \mathfrak{q}(s) d s$ for all $t \in[0, \tilde{h}]$.

Let $E$ be a Banach space, $\beta$ a monotone nonsingular MNC in $E$.

Definition 2.7. A continuous map $\mathfrak{F}: Y \subseteq E \rightarrow E$ is called condensing with respect to a MNC $\beta$ (or $\beta$-condensing) if for every bounded set $\Omega \subseteq Y$ which is not relatively compact, we have

$$
\beta(\mathfrak{F}(\Omega)) \ngtr \beta(\Omega) .
$$

The following fixed point principle (see, e.g., $[17,18]$ ) will be used later.

Theorem 2.8. Let $\mathfrak{W}$ be a bounded convex closed subset of $E$ and $\mathfrak{F}: \mathfrak{W} \rightarrow \mathfrak{W}$ a $\beta$ condensing map. Then Fix $\mathfrak{F}=\{x: x=\mathfrak{F}(x)\}$ is nonempty.

Theorem 2.9. Let $V \subset E$ be a bounded open neighborhood of zero and $\mathfrak{F}: \bar{V} \rightarrow E a$ $\beta$-condensing map satisfying the boundary condition

$$
x \neq \bar{\lambda} \mathfrak{F}(x)
$$

for all $x \in \partial V$ and $0<\bar{\lambda} \leq 1$. Then Fix is a nonempty compact set.

We state a generalization of Gronwall's lemma for singular kernels [[19], Lemma 7.1.1].

Lemma 2.10. Let $x, y:[0, T] \rightarrow[0,+\infty)$ be continuous functions. If $y(\cdot)$ is nondecreasing and there are constants $\mathfrak{a}>0$ and $0<\xi<1$ such that

$$
x(t) \leq y(t)+\mathfrak{a} \int_{0}^{t}(t-s)^{-\xi} x(s) d s
$$

then there exists a constant $\kappa=\kappa(\xi)$ such that

$$
x(t) \leq y(t)+\kappa \mathfrak{a} \int_{0}^{t}(t-s)^{-\xi} \gamma(s) d s, \quad \text { for each } t \in[0, T] .
$$

According to Definitions 2.1-2.3, we can rewrite the nonlocal Cauchy problem (1.1) in the equivalent integral equation

$$
\begin{aligned}
& v(t)=g(v)(0)+\phi(0)+h\left(t, v_{t}\right)-h(0, \phi+g(v))+\frac{1}{\Gamma(q)} \int_{0}^{t}(t-s)^{q-1}\left[A v(s)+f\left(s, v_{s}\right)\right. \\
& +k(v)(s)] d s, \quad t \in[0, T], \\
& v(t)=g(v)(t)+\phi(t), \quad t \in[-r, 0]
\end{aligned}
$$

provided that the integral in (2.1) exists, where

$$
k(v)(t)=\int_{0}^{t} a\left(t, s, v_{s}\right) d s
$$

and $h(0, \varphi+g(v)):=h(0, \varphi(\theta)+g(v)(\theta)), \theta \in[-r, 0]$. 
Set

$$
\begin{array}{ll}
\hat{v}(\lambda)=\int_{0}^{\infty} e^{-\lambda t} v(t) d t, \quad \hat{h}(\lambda)=\int_{0}^{\infty} e^{-\lambda t} h\left(t, v_{t}\right) d t, \\
\hat{f}(\lambda)=\int_{0}^{\infty} e^{-\lambda t} f\left(t, v_{t}\right) d t, \quad \hat{w}(\lambda)=\int_{0}^{\infty} e^{-\lambda t} k(v)(t) d t .
\end{array}
$$

Using the similar method in [1], formally applying the Laplace transform to (2.1), we have

$$
\widehat{v}(\lambda)=\frac{1}{\lambda}(g(v)(0)+\phi(0)-h(0, \phi+g(v)))+\widehat{h}(\lambda)+\frac{1}{\lambda^{q}} A \widehat{v}(\lambda)+\frac{1}{\lambda^{q}}[\widehat{f}(\lambda)+\widehat{w}(\lambda)],
$$

then

$$
\left(\lambda^{q}-A\right) \widehat{v}(\lambda)=\lambda^{q-1}(g(v)(0)+\phi(0)-h(0, \phi+g(v)))+\lambda^{q} \widehat{h}(\lambda)+[\widehat{f}(\lambda)+\widehat{w}(\lambda)]
$$

thus

$$
\begin{aligned}
\hat{v}(\lambda)= & \lambda^{q-1}\left(\lambda^{q}-A\right)^{-1}(g(v)(0)+\phi(0)-h(0, \phi+g(v)))+\lambda^{q}\left(\lambda^{q}-A\right)^{-1} \hat{h}(\lambda) \\
& +\left(\lambda^{q}-A\right)^{-1}[\hat{f}(\lambda)+\hat{w}(\lambda)] \\
= & \lambda^{q-1} \int_{0}^{\infty} e^{-\lambda^{q} s} S(s)(g(v)(0)+\phi(0)-h(0, \phi+g(v))) d s \\
& +\hat{h}(\lambda)+\int_{0}^{\infty} e^{-\lambda^{q} s} A S(s) \hat{h}(\lambda) d s+\int_{0}^{\infty} e^{-\lambda^{q} s} S(s)[\hat{f}(\lambda)+\hat{w}(\lambda)] d s
\end{aligned}
$$

provided that the integral in (2.2) exists.

We consider the one-sided stable probability density in [4] as follows:

$$
\varpi_{q}(\sigma)=\frac{1}{\pi} \sum_{n=1}^{\infty}(-1)^{n-1} \sigma^{-q n-1} \frac{\Gamma(n q+1)}{n !} \sin (n \pi q), \quad \sigma \in(0, \infty),
$$

whose Laplace transform is given by

$$
\int_{0}^{\infty} e^{-\lambda \sigma} \varpi_{q}(\sigma) d \sigma=e^{-\lambda^{q}}, \quad q \in(0,1) \text {. }
$$

Then, using (2.3), we have

$$
\begin{aligned}
& \lambda^{q-1} \int_{0}^{\infty} e^{-\lambda^{q} s} S(s)(g(v)(0)+\phi(0)-h(0, \phi+g(v))) d s \\
= & q \int_{0}^{\infty}(\lambda t)^{q-1} e^{-(\lambda t)^{q}} S\left(t^{q}\right)(g(v)(0)+\phi(0)-h(0, \phi+g(v))) d t \\
= & -\frac{1}{\lambda} \int_{0}^{\infty}\left(\frac{d}{d t} e^{-(\lambda t)^{q}}\right) S\left(t^{q}\right)(g(v)(0)+\phi(0)-h(0, \phi+g(v))) d t \\
= & \int_{0}^{\infty} \int_{0}^{\infty} e^{-\lambda t \sigma} \sigma \varpi_{q}(\sigma) S\left(t^{q}\right)(g(v)(0)+\phi(0)-h(0, \phi+g(v))) d \sigma d t \\
= & \int_{0}^{\infty} e^{-\lambda t}\left[\int_{0}^{\infty} \varpi_{q}(\sigma) S\left(\frac{t^{q}}{\sigma^{q}}\right)(g(v)(0)+\phi(0)-h(0, \phi+g(v))) d \sigma\right] d t
\end{aligned}
$$


and

$$
\begin{aligned}
& \int_{0}^{\infty} e^{-\lambda^{q} s} S(s) \hat{f}(\lambda) d s \\
= & \int_{0}^{\infty} e^{-(\lambda \tau)^{q}} q \tau^{q-1} S\left(\tau^{q}\right)\left(\int_{0}^{\infty} e^{-\lambda t} f\left(t, v_{t}\right) d t\right) d \tau \\
= & \int_{0}^{\infty} \int_{0}^{\infty} e^{-\lambda \tau \sigma} q \tau^{q-1} \varpi_{q}(\sigma) S\left(\tau^{q}\right)\left(\int_{0}^{\infty} e^{-\lambda t} f\left(t, v_{t}\right) d t\right) d \sigma d \tau \\
= & q \int_{0}^{\infty} \int_{0}^{\infty} e^{-\lambda \theta} \frac{\theta^{q-1}}{\sigma^{q}} \varpi_{q}(\sigma) S\left(\frac{\theta^{q}}{\sigma^{q}}\right)\left(\int_{0}^{\infty} e^{-\lambda t} f\left(t, v_{t}\right) d t\right) d \sigma d \theta \\
= & q \int_{0}^{\infty}\left(\int_{0}^{\infty} \int_{t}^{\infty} e^{-\lambda \tau} \frac{(\tau-t)^{q-1}}{\sigma^{q}} \varpi_{q}(\sigma) S\left(\frac{(\tau-t)^{q}}{\sigma^{q}}\right) f\left(t, v_{t}\right) d \tau d t\right) d \sigma \\
= & q \int_{0}^{\infty}\left(\int_{0}^{\infty} \int_{0}^{\tau} e^{-\lambda \tau} \frac{(\tau-t)^{q-1}}{\sigma^{q}} \varpi_{q}(\sigma) S\left(\frac{(\tau-t)^{q}}{\sigma^{q}}\right) f\left(t, v_{t}\right) d t d \tau\right) d \sigma \\
= & \int_{0}^{\infty} e^{-\lambda t}\left[\int_{0}^{t} \int_{0}^{\infty} \frac{(t-t)^{q-1}}{\sigma^{q}} \varpi_{q}(\sigma) S\left(\frac{(t-s)^{q}}{\sigma^{q}}\right) f\left(s, v_{s}\right) d \sigma d s\right] d t .
\end{aligned}
$$

Similarly, we have

$$
\begin{aligned}
& \int_{0}^{\infty} e^{-\lambda^{q} s} S(s) \hat{w}(\lambda) d s \\
= & \int_{0}^{\infty} e^{-\lambda t}\left[q \int_{0}^{t} \int_{0}^{\infty} \frac{(t-s)^{q-1}}{\sigma^{q}} \varpi_{q}(\sigma) S\left(\frac{(t-s)^{q}}{\sigma^{q}}\right) k(v)(s) d \sigma d s\right] d t
\end{aligned}
$$

and

$$
\begin{aligned}
& \int_{0}^{\infty} e^{-\lambda^{q} s} A S(s) \hat{h}(\lambda) d s \\
= & \int_{0}^{\infty} e^{-\lambda t}\left[q \int_{0}^{t} \int_{0}^{\infty} \frac{(t-s)^{q-1}}{\sigma^{q}} \varpi_{q}(\sigma) A S\left(\frac{(t-s)^{q}}{\sigma^{q}}\right) h\left(s, v_{s}\right) d \sigma d s\right] d t .
\end{aligned}
$$

Thus, from (2.4)-(2.7), we obtain

$$
\begin{aligned}
\hat{v}(\lambda) & =\int_{0}^{\infty} e^{-\lambda t}\left[\int_{0}^{\infty} \varpi_{q}(\sigma) S\left(\frac{t^{q}}{\sigma^{q}}\right)(g(v)(0)+\phi(0)-h(0, \phi+g(v))) d \sigma+h\left(t, v_{t}\right)\right. \\
& +q \int_{0}^{t} \int_{0}^{\infty} \frac{(t-s)^{q-1}}{\sigma^{q}} \varpi_{q}(\sigma) A S\left(\frac{(t-s)^{q}}{\sigma^{q}}\right) h\left(s, v_{s}\right) d \sigma d s \\
& +q \int_{0}^{t} \int_{0}^{\infty} \frac{(t-s)^{q-1}}{\sigma^{q}} \varpi_{q}(\sigma) S\left(\frac{(t-s)^{q}}{\sigma^{q}}\right) f\left(s, v_{s}\right) d \sigma d s \\
& \left.+q \int_{0}^{t} \int_{0}^{\infty} \frac{(t-s)^{q-1}}{\sigma^{q}} \varpi_{q}(\sigma) S\left(\frac{(t-s)^{q}}{\sigma^{q}}\right) k(v)(s) d \sigma d s\right] d t .
\end{aligned}
$$


We invert the last Laplace transform to obtain

$$
\begin{aligned}
v(t)= & \int_{0}^{\infty} \varpi_{q}(\sigma) S\left(\frac{t^{q}}{\sigma^{q}}\right)(g(v)(0)+\phi(0)-h(0, \phi+g(v))) d \sigma+h\left(t, v_{t}\right) \\
& +q \int_{0}^{t} \int_{0}^{\infty} \frac{(t-s)^{q-1}}{\sigma^{q}} \varpi_{q}(\sigma) A S\left(\frac{(t-s)^{q}}{\sigma^{q}}\right) h\left(s, v_{s}\right) d \sigma d s \\
& +q \int_{0}^{t} \int_{0}^{\infty} \frac{(t-s)^{q-1}}{\sigma^{q}} \varpi_{q}(\sigma) S\left(\frac{(t-s)^{q}}{\sigma^{q}}\right) f\left(s, v_{s}\right) d \sigma d s \\
& +q \int_{0}^{t} \int_{0}^{\infty} \frac{(t-s)^{q-1}}{\sigma^{q}} \varpi_{q}(\sigma) S\left(\frac{(t-s)^{q}}{\sigma^{q}}\right) k(v)(s) d \sigma d s \\
= & \int_{0}^{\infty} \xi_{q}(\sigma) S\left(t^{q} \sigma\right)(g(v)(0)+\phi(0)-h(0, \phi+g(v))) d \sigma+h\left(t, v_{t}\right) \\
& +q \int_{0}^{t} \int_{0}^{\infty} \sigma(t-s)^{q-1} \xi_{q}(\sigma) A S\left((t-s)^{q} \sigma\right) h\left(s, v_{s}\right) d \sigma d s \\
& +q \int_{0}^{t} \int_{0}^{\infty} \sigma(t-s)^{q-1} \xi_{q}(\sigma) S\left((t-s)^{q} \sigma\right) f\left(s, v_{s}\right) d \sigma d s \\
& +q \int_{0}^{t} \int_{0}^{\infty} \sigma(t-s)^{q-1} \xi_{q}(\sigma) S\left((t-s)^{q} \sigma\right) k(v)(s) d \sigma d s,
\end{aligned}
$$

where $\xi_{q}$ is a probability density function defined on $(0, \infty)$ such that

$$
\xi_{q}(\sigma)=\frac{1}{q} \sigma^{-1-\frac{1}{q}} \varpi_{q}\left(\sigma^{-\frac{1}{q}}\right) \geq 0 .
$$

For any $z \in X$, we define operators $\{Q(t)\}_{t \geq 0}$ and $\{R(t)\}_{t \geq 0}$ by

$$
\begin{aligned}
& Q(t) z=\int_{0}^{\infty} \xi_{q}(\sigma) S\left(t^{q} \sigma\right) z d \sigma, \\
& R(t) z=q \int_{0}^{\infty} \sigma t^{q-1} \xi_{q}(\sigma) S\left(t^{q} \sigma\right) z d \sigma .
\end{aligned}
$$

Then from above induction, we can give the following definition of the mild solution of (1.1).

Definition 2.11. A continuous function $v:[-r, T] \rightarrow X$ is a mild solution of $(1.1)$ if the function $v$ satisfies the equation

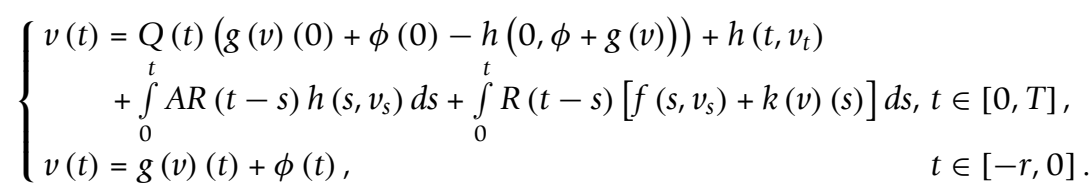


Remark 2.12. (i) Noting that $\int_{0}^{\infty} \xi_{q}(\sigma) d \sigma=1$, we obtain

$$
\|Q(t)\| \leq M .
$$

(ii) According to [4], direct calculation gives that

$$
\int_{0}^{\infty} \sigma^{v} \xi_{q}(\sigma) d \sigma=\int_{0}^{\infty} \frac{1}{\sigma^{q \nu}} \varpi_{q}(\sigma) d \sigma=\frac{\Gamma(1+v)}{\Gamma(1+q v)}, \quad v \in(0,1],
$$

then, we can obtain

$$
\|R(t)\| \leq \frac{q M}{\Gamma(1+q)} t^{q-1}, \quad t>0 .
$$

(iii) For any $\alpha \in(0,1)$, we have

$$
\left\|(-A)^{\alpha} R(t)\right\| \leq q t^{q(1-\alpha)-1} \frac{C_{\alpha} \Gamma(2-\alpha)}{\Gamma(1+q(1-\alpha))}, \quad t>0 .
$$

Indeed, for any $z \in X$ we can see that

$$
\begin{aligned}
\left\|(-A)^{\alpha} R(t) z\right\| & =\left\|q \int_{0}^{\infty} \sigma t^{q-1} \xi_{q}(\sigma)(-A)^{\alpha} S\left(t^{q} \sigma\right) z d \sigma\right\| \\
& \leq q t^{q-1} \int_{0}^{\infty} \sigma \xi_{q}(\sigma) \frac{C_{\alpha}}{\left(t^{q} \sigma\right)^{\alpha}} d \sigma\|z\| \\
& =q t^{q(1-\alpha)-1} \frac{C_{\alpha} \Gamma(2-\alpha)}{\Gamma(1+q(1-\alpha))}\|z\| .
\end{aligned}
$$

\section{Main results}

We will require the following assumptions.

(Hf) (1) $f:[0, T] \times C([-r, 0], X) \rightarrow X$ satisfies $f(\cdot, w):[0, T] \rightarrow X$ is measurable for all $w \in C([-r, 0], X)$ and $f(t, \cdot): C([-r, 0], X) \rightarrow X$ is continuous for a.e. $t \in[0, T]$, and there exists a positive function $\mu(\cdot) \in L^{p}\left([0, T], \mathrm{R}^{+}\right)\left(p>\frac{1}{q \alpha}>1\right)$ such that

$$
\|f(t, w)\| \leq \mu(t)\|w\|_{[-r, 0]}
$$

for almost all $t \in[0, T]$.

(2) There exists a nondecreasing function $\eta \in L^{p}\left([0, T], \mathbf{R}^{+}\right)$such that for any bounded set $D \subset C([-r, 0], X)$,

$$
\chi(f(t, D)) \leq \eta(t) \sup _{\theta \in[-r, 0]} \chi(D(\theta)), \quad \text { a.e. } \quad t \in[0, T] .
$$

(Hh) (1) $h:[0, T] \times C([-r, 0], X) \rightarrow X$ is continuous and there exists constants $\alpha \in(0$, $1)$ and $M_{1}, L_{h}>0$ such that $h \in D\left((-A)^{\alpha}\right)$ and for any $\phi \in C([-r, 0], X)$ the function $(-A)$ ${ }^{\alpha} h(\cdot, \phi)$ is strongly measurable and satisfies

$$
\left\|(-A)^{\alpha} h(t, \varphi)\right\| \leq M_{1}\left(\|\varphi\|_{[-r, 0]}+1\right),
$$


and for $t_{1}, t_{2} \in[0, T], \varphi, \tilde{\varphi} \in C([-r, 0], X)$,

$$
\left\|(-A)^{\alpha} h\left(t_{1}, \varphi\right)-(-A)^{\alpha} h\left(t_{2}, \tilde{\varphi}\right)\right\| \leq L_{h}\left(\left|t_{1}-t_{2}\right|+\|\varphi-\tilde{\varphi}\|_{[-r, 0]}\right) .
$$

(2) There exists $M_{2}>0$ such that for any bounded set $D \subset C([-r, 0], X)$,

$$
\chi\left((-A)^{\alpha} h(t, D)\right) \leq M_{2} \sup _{\theta \in[-r, 0]} \chi(D(\theta)), \quad \text { a.e. } \quad t \in[0, T] .
$$

(Ha) (1) $a: \Delta \times C([-r, 0], X) \rightarrow X$ and $a(t, s, \cdot): C([-r, 0], X) \rightarrow X$ is continuous for a.e. $(t, s) \in \Delta$, and for each $w \in C([-r, 0], X)$, the function $a(\cdot,, w): \Delta \rightarrow X$ is measurable. Moreover, there exists a function $m: \Delta \rightarrow \mathbf{R}^{+}$with $\sup _{t \in[0, T]} \int_{0}^{t} m(t, s) d s:=m^{*}<\infty$ such that

$$
\|a(t, s, w)\| \leq m(t, s)\|w\|_{[-r, 0]}
$$

for almost all $(t, s) \mathrm{L} \Delta$.

(2) For any bounded set $D \subset C([-r, 0], X)$ and $0 \leq s \leq t \leq T$, there exists a function $\zeta$ : $\Delta \rightarrow \mathbf{R}^{+}$such that

$$
\chi(a(t, s, D)) \leq \zeta(t, s) \sup _{\theta \in[-r, 0]} \chi(D(\theta)),
$$

where $\sup _{t \in[0, T]} \int_{0}^{t} \zeta(t, s) d s:=\zeta^{*}<\infty$

(Hg) (1) There exists a continuous function $L_{g}:[-r, 0] \rightarrow \mathbf{R}^{+}$such that

$$
\left\|g\left(v_{1}\right)(t)-g\left(v_{2}\right)(t)\right\| \leq L_{g}(t)\left\|v_{1}(t)-v_{2}(t)\right\|, \quad t \in[-r, 0] .
$$

(2) The function $g(v)(\cdot):[-r, 0] \rightarrow C([-r, 0], X)$ is equicontinuous and uniformly bounded, that is, there exists a constant $N>0$ such that

$$
\|g(v)\|_{[-r, 0]} \leq N \text { for all } v \in C([-r, 0], X) .
$$

Theorem 3.1. Assume that (Hf), (Hh), (Ha) and $(\mathrm{Hg})$ are satisfied, if

$$
\text { (1) } M_{0} \cdot \max \left\{M_{1}, L_{h}\right\}+\frac{q M}{\Gamma(1+q)} l_{p, q} T^{q-\frac{1}{p}}\|\mu\|_{L^{p}}<1 \text {, }
$$

(2) $L_{g}^{*}\left(1+M M_{0} M_{2}+M\right)+M_{0} M_{2}<1$,

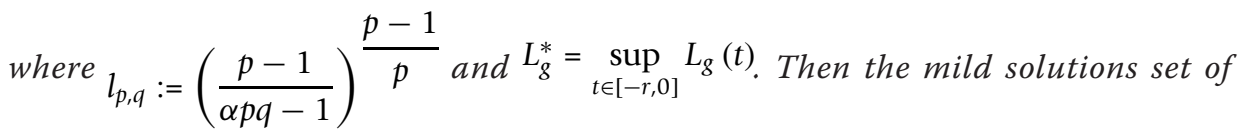
problem (1.1) is a nonempty compact subset of the space $C([-r, T], X)$.

Proof. Define the operator $\mathcal{F}: C([-r, T], X) \rightarrow C([-r, T], X)$ in the following way:

$$
(\mathcal{F} v)(t)=\left\{\begin{array}{l}
g(v)(t)+\phi(t), \\
Q(t)(g(v)(0)+\phi(0)-h(0, \phi+g(v)))+h\left(t, v_{t}\right) \\
+\int_{0}^{t} A R(t-s) h\left(s, v_{s}\right) d s+\int_{0}^{t} R(t-s)\left[f\left(s, v_{s}\right)+k(v)(s)\right] d s, t \in[0, T]
\end{array}\right.
$$

It is clear that the operator $\mathcal{F}$ is well defined.

The operator $\mathcal{F}$ can be written in the form $\mathcal{F}=\sum_{i=1}^{2} \mathcal{F}_{i}$, where the operators $\mathcal{F}_{i}, i=1,2$ are defined as follows: 


$$
\begin{aligned}
& \left(\mathcal{F}_{1} v\right)(t)= \begin{cases}g(v)(t)+\phi(t), & t \in[-r, 0], \\
Q(t)(g(v)(0)+\phi(0)-h(0, \phi+g(v)))+h\left(t, v_{t}\right), & t \in[0, T],\end{cases} \\
& \left(\mathcal{F}_{2} v\right)(t)= \begin{cases}0, & t \in[-r, 0], \\
\int_{0}^{t} A R(t-s) h\left(s, v_{s}\right) d s+\int_{0}^{t} R(t-s)\left[f\left(s, v_{s}\right)+k(v)(s)\right] d s, & t \in[0, T] .\end{cases}
\end{aligned}
$$

Let $\left\{v^{n}\right\}_{n \in \mathbf{N}}$ be a sequence such that $v^{n} \rightarrow v$ in $C([-r, T], X)$ as $n \rightarrow \infty$. By the continuity of $g$ and $h$, we can see that $\mathcal{F}_{1}$ is continuous.

Moreover, noting (2.10), we have

$$
\begin{aligned}
& \left\|\int_{0}^{t} A R(t-s) h\left(s, v_{s}^{n}\right) d s-\int_{0}^{t} A R(t-s) h\left(s, v_{s}\right) d s\right\| \\
\leq & \int_{0}^{t}\left\|(-A)^{1-\alpha} R(t-s)\right\|\left\|(-A)^{\alpha} h\left(s, v_{s}^{n}\right)-(-A)^{\alpha} h\left(s, v_{s}\right)\right\| d s \\
\leq & \frac{q C_{1-\alpha} \Gamma(1+\alpha)}{\Gamma(1+q \alpha)} L_{h}\left\|v^{n}-v\right\|_{[-r, T]} \int_{0}^{t}(t-s)^{q \alpha-1} d s \\
\leq & \frac{C_{1-\alpha} \Gamma(1+\alpha)}{\alpha \Gamma(1+q \alpha)} L_{h} T^{q \alpha}\left\|v^{n}-v\right\|_{[-r, T]} \rightarrow 0, \quad \text { as } n \rightarrow \infty .
\end{aligned}
$$

Since $f$ satisfies (Hf)(1) and $a$ satisfies (Ha)(1), for almost every $t \in[0, T]$ and $(t, s) \in$ $\Delta$, we get

$$
\begin{aligned}
f\left(t, v_{t}^{n}\right) & \rightarrow f\left(t, v_{t}\right), \quad \text { as } n \rightarrow \infty, \\
a\left(t, s, v_{s}^{n}\right) & \rightarrow \mathrm{a}\left(t, s, v_{s}\right), \quad \text { as } n \rightarrow \infty .
\end{aligned}
$$

Noting that $v^{n} \rightarrow v$ in $C([-r, T], X)$, we can see that there exists $\varepsilon>0$ such that $\| v^{n}$ $v \|_{[-r, T]} \leq \varepsilon$ for $n$ sufficiently large. Therefore, we have

$$
\begin{aligned}
\left\|f\left(t, v_{t}^{n}\right)-f\left(t, v_{t}\right)\right\| & \leq \mu(t)\left\|v_{t}^{n}\right\|_{[-r, 0]}+\mu(t)\left\|v_{t}\right\|_{[-r, 0]} \\
& \leq \mu(t)\left\|v_{t}^{n}-v_{t}\right\|_{[-r, 0]}+2 \mu(t)\left\|v_{t}\right\|_{[-r, 0]} \\
& \leq \mu(t)\left(\varepsilon+2\|v\|_{[-r, T]}\right)
\end{aligned}
$$

and similarly,

$$
\begin{aligned}
\left\|a\left(t, s, v_{s}^{n}\right)-a\left(t, s, v_{s}\right)\right\| & \leq m(t, s)\left(\varepsilon+2\|v\|_{[-r, T]}\right), \\
\left\|\int_{0}^{t} a\left(t, s, v_{s}^{n}\right) d s-\int_{0}^{t} a\left(t, s, v_{s}\right) d s\right\| & \leq m^{*}\left(\varepsilon+2\|v\|_{[-r, T]}\right) .
\end{aligned}
$$

It follows from the Lebesgue's dominated convergence theorem that

$$
\left\|\int_{0}^{t} a\left(t, s, v_{s}^{n}\right) d s-\int_{0}^{t} a\left(t, s, v_{s}\right) d s\right\| \rightarrow 0, \quad \text { as } n \rightarrow \infty,
$$


and

$$
\begin{aligned}
& \left\|\int_{0}^{t} R(t-s)\left\{f\left(s, v_{s}\right)^{n}+\int_{0}^{s} a\left(s, \tau, v_{\tau}^{n}\right) d \tau-\left[f\left(s, v_{s}+\int_{0}^{s} a\left(s, \tau, v_{\tau}\right) d \tau\right]\right\} d s\right]\right\| \\
& \leq \frac{q M}{\Gamma(1+q)} \int_{0}^{t}(t-s)^{q-1}\left[\| f\left(s, v_{s}\right)^{n}-f\left(s, v_{s}\left\|+\int_{0}^{s}\right\| a\left(s, \tau, v_{\tau}^{n}\right)-a\left(s, \tau, v_{\tau}\right) \| d \tau\right] d s\right. \\
& \rightarrow 0, \quad \text { as } n \rightarrow \infty .
\end{aligned}
$$

Therefore, we obtain that

$$
\lim _{n \rightarrow \infty}\left\|\mathcal{F}_{2} v^{n}-\mathcal{F}_{2} v\right\|_{[-r, T]}=0 .
$$

Now, from (3.3), we can see that $\mathcal{F}$ is continuous.

Let $\chi$ be a Hausdorff $\mathrm{MNC}$ in $X$, we consider the measure of noncompactness $\beta$ in the space $C([-r, T], X)$ with values in the cone $\mathbf{R}_{+}^{2}$ of the following way: for every bounded subset $\Omega \subset C([-r, T], X)$,

$$
\beta(\Omega)=\left(\Psi(\Omega), \bmod _{c}(\Omega)\right)
$$

where $\bmod _{c}(\Omega)$ is the module of equicontinuity of $\Omega$ given by:

$$
\begin{aligned}
\bmod _{c}(\Omega) & =\lim _{\delta \rightarrow 0} \sup _{v \in \Omega} \max _{\left|t_{1}-t_{2}\right| \leq \delta}\left\|v\left(t_{1}\right)-v\left(t_{2}\right)\right\|, \\
\Psi(\Omega) & =\sup _{t \in[-r, 0]} \chi(\Omega(t))+\sup _{t \in[0, T]}\left(e^{-L t} \sup _{s \in[0, t]} \chi(\Omega(s))\right),
\end{aligned}
$$

where $L>0$ is a constant chosen so that

$$
\begin{aligned}
& \frac{q C_{1-\alpha} \Gamma(1+\alpha)}{\Gamma(1+q \alpha)} \max \left\{M_{1}, M_{2}\right\} \sup _{t \in[0, T]} \int_{0}^{t}(t-s)^{q \alpha-1} e^{-L(t-s)} d s=L_{1}<1, \\
& \frac{q M}{\Gamma(1+q)} \sup _{t \in[0, T]} \int_{0}^{t}(t-s)^{q-1}\left(\eta(s)+\zeta^{*}\right) e^{-L(t-s)} d s=L_{2}<1, \\
& \frac{q M}{\Gamma(1+q)} \sup _{t \in[0, T]} \int_{0}^{t}(t-s)^{q-1}\left(\mu(s)+m^{*}\right) e^{-L(t-s)} d s=L_{3}<1 .
\end{aligned}
$$

Noting that for any $\gamma \in L^{1}([0, T], X)$, we have

$$
\lim _{L \rightarrow+\infty} \sup _{t \in[0, T]} \int_{0}^{t} e^{-L(t-s)} \gamma(s) d s=0,
$$

so, we can take the appropriate $L$ to satisfy (3.4)-(3.6).

Next, we show that the operator $\mathcal{F}$ is $\beta$-condensing on every bounded subset of $C$ $([-r, T], X)$.

Let $\Omega \subset C([-r, T], X)$ be a nonempty, bounded set such that

$$
\beta(\mathcal{F}(\Omega)) \geq \beta(\Omega)
$$


Firstly, we estimate $\Psi(\Omega)$. For $t \in[-r, 0], v_{1}, v_{2} \in \Omega$, we have

$$
\left\|g\left(v_{1}\right)(t)-g\left(v_{2}\right)(t)\right\| \leq L_{g}^{*}\left\|v_{1}(t)-v_{2}(t)\right\|,
$$

then

$$
\sup _{t \in[-r, 0]} \chi(g(\Omega)(t)) \leq L_{g}^{*} \sup _{t \in[-r, 0]} \chi(\Omega(t)),
$$

therefore,

$$
\sup _{t \in[-r, 0]} \chi\left(\left(\mathcal{F}_{1} \Omega\right)(t)\right)=\sup _{t \in[-r, 0]} \chi(g(\Omega)(t)) \leq L_{g}^{*} \sup _{t \in[-r, 0]} \chi(\Omega(t)) .
$$

For $t \in[0, T]$, one gets

$$
\|Q(t)\|\left\|g\left(v_{1}\right)(0)-g\left(v_{2}\right)(0)\right\| \leq M L_{g}^{*}\left\|v_{1}(0)-v_{2}(0)\right\|,
$$

thus, we have

$$
\chi(Q(t) g(\Omega)(0)) \leq M L_{g}^{*} \chi(\Omega(0)) \leq M L_{g}^{*} \Psi(\Omega) .
$$

Moreover, we see that

$$
\begin{aligned}
& \chi(-Q(t) h(0, \phi+g(\Omega)) \\
\leq & M \cdot \chi\left((-A)^{-\alpha}(-A)^{\alpha} h(0, \phi+g(\Omega))\right) \\
\leq & M M_{0} M_{2} \sup _{s \in[-r, 0]} \chi(g(\Omega)(s)) \\
\leq & M M_{0} M_{2} L_{g}^{*} \sup _{s \in[-r, 0]} \chi(\Omega(s)) \\
\leq & M M_{0} M_{2} L_{g}^{*} \Psi(\Omega) .
\end{aligned}
$$

For $t \in[0, T]$, noting that

$$
\begin{aligned}
\sup _{\theta \in[-r, 0]} \chi(\Omega(t+\theta)) & =\sup _{s \in[t-r, t]} \chi(\Omega(s)) \\
& \leq \sup _{s \in[-r, 0]} \chi(\Omega(s))+\sup _{s \in[0, t]} \chi(\Omega(s)) \\
& \leq e^{L t}\left(\sup _{s \in[-r, 0]} \chi(\Omega(s))+e^{-L t} \sup _{s \in[0, t]} \chi(\Omega(s))\right) \\
& \leq e^{L t} \Psi(\Omega),
\end{aligned}
$$

then we have

$$
\begin{aligned}
\chi\left(h\left(t, \Omega_{t}\right)\right) & =\chi\left((-A)^{-\alpha}(-A)^{\alpha} h\left(t, \Omega_{t}\right)\right) \\
& \leq M_{0} M_{2} \sup _{\theta \in[-r, 0]} \chi(\Omega(t+\theta)) \\
& \leq M_{0} M_{2} e^{L t} \Psi(\Omega),
\end{aligned}
$$

where $\Omega_{t}=\left\{v_{t}: v \in \Omega\right\}$. Therefore

$$
\sup _{t \in[0, T]}\left(e^{-L t} \sup _{s \in[0, t]} \chi\left(h\left(s, \Omega_{s}\right)\right)\right) \leq M_{0} M_{2} \Psi(\Omega) .
$$


Now, from (3.8)-(3.10), and (3.12), we can see

$$
\begin{aligned}
\Psi\left(\mathcal{F}_{1} \Omega\right) & =\sup _{t \in[-r, 0]} \chi\left(\left(\mathcal{F}_{1} \Omega\right)(t)\right)+\sup _{t \in[0, T]}\left(e^{-L t} \sup _{s \in[0, t]} \chi\left(\left(\mathcal{F}_{1} \Omega\right)(s)\right)\right) \\
& \leq L_{g}^{*} \sup _{t \in[-r, 0]} \chi(\Omega(t))+\left(M M_{0} M_{2} L_{g}^{*}+M L_{g}^{*}+M_{0} M_{2}\right) \Psi(\Omega) \\
& \leq\left[L_{g}^{*}\left(1+M M_{0} M_{2}+M\right)+M_{0} M_{2}\right] \Psi(\Omega) .
\end{aligned}
$$

For any $t \in[0, T]$, we set

$$
\Lambda_{1}(\Omega)(t)=\left\{\int_{0}^{t} A R(t-s) h\left(s, v_{s}\right) d s: v \in \Omega\right\} .
$$

We consider the multifunction $s \in[0, t] \otimes G(s)$,

$$
G(s)=\left\{A R(t-s) h\left(s, v_{s}\right): v \in \Omega\right\} .
$$

Obviously, $G$ is integrable, and from $(\mathrm{Hh})(1)$ it follows that it is integrably bounded. Moreover, noting that $(\mathrm{Hh})(2)$ and (3.11), we have the following estimate for a.e. $s \in$ $[0, t]$ :

$$
\begin{aligned}
\chi(G(s)) & =\chi\left(\left\{(-A)^{1-\alpha} R(t-s)(-A)^{\alpha} h\left(s, v_{s}\right): v \in \Omega\right\}\right) \\
& =\chi\left((-A)^{1-\alpha} R(t-s)(-A)^{\alpha} h\left(s, \Omega_{s}\right)\right) \\
& \leq \frac{q C_{1-\alpha} \Gamma(1+\alpha)}{\Gamma(1+q \alpha)}(t-s)^{q \alpha-1} M_{2} \sup _{\theta \in[-r, 0]} \chi(\Omega(s+\theta)) \\
& \leq \frac{q C_{1-\alpha} \Gamma(1+\alpha)}{\Gamma(1+q \alpha)}(t-s)^{q \alpha-1} M_{2} e^{L s} \Psi(\Omega)
\end{aligned}
$$

Applying Proposition 2.6, we have

$$
\chi\left(\Lambda_{1}(\Omega)(t)\right)=\chi\left(\int_{0}^{t} G(s) d s\right) \leq \frac{q C_{1-\alpha} \Gamma(1+\alpha)}{\Gamma(1+q \alpha)} M_{2} \int_{0}^{t}(t-s)^{q \alpha-1} e^{L s} d s \cdot \Psi(\Omega) .
$$

Therefore,

$$
\begin{aligned}
& \sup _{t \in[0, T]}\left(e^{-L t} \sup _{s \in[0, t]} \chi\left(\Lambda_{1}(\Omega)(s)\right)\right) \\
\leq & \frac{q C_{1-\alpha} \Gamma(1+\alpha)}{\Gamma(1+q \alpha)} M_{2} \sup _{t \in[0, T]} \int_{0}^{t}(t-s)^{q \alpha-1} e^{-L(t-s)} d s \cdot \Psi(\Omega) \\
= & L_{1} \Psi(\Omega) .
\end{aligned}
$$

Similar to above induction, if we set

$$
\Lambda_{2}(\Omega)(t)=\left\{\int_{0}^{t} R(t-s)\left[f\left(s, v_{s}\right)+k(v)(s)\right] d s: v \in \Omega\right\}
$$

then we can see that the multifunction $s \in[0, t] \multimap \widetilde{G}(s)$,

$$
\tilde{G}(s)=\left\{R(t-s)\left[f\left(s, v_{s}\right)+k(v)(s)\right]: v \in \Omega\right\}
$$


is integrable, and from (Hf)(1), (Ha)(1) it follows that it is integrably bounded. Moreover, noting that $(\mathrm{Hf})(2),(\mathrm{Ha})(2)$, Proposition 2.6 and (3.11), we have the following estimate for a.e. $s \in[0, t]$ :

$$
\chi(\widetilde{G}(s)) \leq \frac{q M}{\Gamma(1+q)}(t-s)^{q-1}\left[\eta(s)+\zeta^{*}\right] \cdot e^{L s} \Psi(\Omega),
$$

furthermore,

$$
\begin{aligned}
\sup _{t \in[0, T]}\left(e^{-L t} \sup _{s \in[0, t]} \chi\left(\Lambda_{2}(\Omega)(s)\right)\right) & \leq \frac{q M}{\Gamma(1+q)} \sup _{t \in[0, T]} \int_{0}^{t}(t-s)^{q-1}\left[\eta(s)+\zeta^{*}\right] e^{-L(t-s)} d s \cdot \Psi(\Omega) \\
& =L_{2} \Psi(\Omega) .
\end{aligned}
$$

Now, it follows from (3.14) and (3.15) that $\Psi\left(\mathcal{F}_{2} \Omega\right) \leq \sum_{i=1}^{2} L_{i} \Psi(\Omega)$. Then, noting (3.13) and choose $L_{i}(i=1,2)$ such that

$$
\Psi(\mathcal{F} \Omega) \leq\left[L_{g}^{*}\left(1+M M_{0} M_{2}+M\right)+M_{0} M_{2}+\sum_{i=1}^{2} L_{i}\right] \Psi(\Omega) \leq \widetilde{L} \Psi(\Omega),
$$

where $0<\widetilde{L}<1$.

From (3.7), we have $\Psi(\Omega)=0$. Next, we will prove $\bmod _{c}(\Omega)=0$.

Let $\delta>0, t_{1}, t_{2} \in[0, T]$ such that $0<\left|t_{1}-t_{2}\right| \leq \delta$ and $v \in \Omega$, we obtain

$$
\begin{aligned}
\left\|h\left(t_{1}, v_{t_{1}}\right)-h\left(t_{1}, v_{t_{2}}\right)\right\| & \leq\left\|(-A)^{-\alpha}\right\|\left\|(-A)^{\alpha}\left(h\left(t_{1}, v_{t_{1}}\right)-h\left(t_{2}, v_{t_{2}}\right)\right)\right\| \\
& \leq M_{0} L_{h}\left(\left|t_{1}-t_{2}\right|+\left\|v_{t_{1}}-v_{t_{2}}\right\|_{[-r, 0]}\right) \\
& =M_{0} L_{h}\left(\left|t_{1}-t_{2}\right|+\sup _{\theta \in[-r, 0],\left|t_{1}-t_{2}\right| \leq \delta}\left\|v\left(t_{1}+\theta\right)-v\left(t_{2}+\theta\right)\right\|\right) \\
& \leq M_{0} L_{h}\left(\left|t_{1}-t_{2}\right|+\sup _{s_{1}, s_{2} \in[-r, T],\left|s_{1}-s_{2}\right| \leq \delta}\left\|v\left(s_{1}\right)-v\left(s_{2}\right)\right\|\right) .
\end{aligned}
$$

Moreover, noting that $(\mathrm{Hg})(2)$ and the continuity of $S(t)$ in the uniform operator topology for $t>0$ we have

$$
\bmod _{c}\left(\mathcal{F}_{1} \Omega\right) \leq M_{0} L_{h} \bmod _{c}(\Omega) .
$$

For $0<t_{2}<t_{1} \leq T$ and $v \in \Omega$, we have

$$
\begin{aligned}
& \quad\left\|\int_{0}^{t_{1}} A R\left(t_{1}-s\right) h\left(s, v_{s}\right) d s-\int_{0}^{t_{2}} A R\left(t_{2}-s\right) h\left(s, v_{s}\right) d s\right\| \\
& \leq \int_{0}^{t_{2}}\left\|A\left(R\left(t_{1}-s\right)-R\left(t_{2}-s\right)\right) h\left(s, v_{s}\right)\right\| d s+\int_{t_{2}}^{t_{1}}\left\|(-A)^{1-\alpha} R\left(t_{1}-s\right)\right\|\left\|(-A)^{\alpha} h\left(s, v_{s}\right)\right\| d s \\
& \leq q \int_{0}^{t_{2}} \int_{0}^{\infty} \sigma\left\|\left[\left(t_{1}-s\right)^{q-1}-\left(t_{2}-s\right)^{q-1}\right] \xi_{q}(\sigma) A S\left(\left(t_{1}-s\right)^{q} \sigma\right) h\left(s, v_{s}\right)\right\| d \sigma d s \\
& \quad+q \int_{0}^{t_{2}} \int_{0}^{\infty} \sigma\left(t_{2}-s\right)^{q-1} \xi_{q}(\sigma)\left\|A\left(S\left(\left(t_{1}-s\right)^{q} \sigma\right)-S\left(\left(t_{2}-s\right)^{q} \sigma\right)\right) h\left(s, v_{s}\right)\right\| d \sigma d s \\
& \quad+M_{1}\left(\|v\|_{[-r, T]}+1\right)\left(t_{1}-t_{2}\right)^{q \alpha} \frac{C_{1-\alpha} \Gamma(1+\alpha)}{\alpha \Gamma(1+q \alpha)} \\
& =I_{1}+I_{2}+I_{3} .
\end{aligned}
$$

Noting that $s \rightarrow A S\left(\left(t_{1}-s\right)^{q} \sigma\right) h\left(s, v_{s}\right)$ is integrable on $\left[0, t_{1}\right)$, then $I_{1} \rightarrow 0$, as $t_{2} \rightarrow t_{1}$. In view of the fact that $\{S(t)\}_{t \geq 0}$ is an analytic semigroup, then for $s \in[0, t), \sigma \in(0$, $\infty)$, the operator function $s \rightarrow A S\left((t-s)^{q} \sigma\right)$ is continuous in the uniform operator 
topology in $[0, t)$ and noting that $s \rightarrow h\left(s, v_{s}\right)$ is integrable on $[0, t], I_{2} \rightarrow 0$, as $t_{2} \rightarrow t_{1}$. Obviously $\mathrm{I}_{3} \rightarrow 0$, as $t_{2} \rightarrow t_{1}$.

Moreover, nothing that

$$
\left\|f\left(s, v_{s}\right)\right\|+\|k(v)(s)\| \leq\left(\mu(s)+m^{*}\right)\|v\|_{[-r, T]},
$$

we have

$$
\begin{aligned}
& \left\|\int_{0}^{t_{1}} R\left(t_{1}-s\right)\left[f\left(s, v_{s}\right)+k(v)(s)\right] d s-\int_{0}^{t_{2}} R\left(t_{2}-s\right)\left[f\left(s, v_{s}\right)+k(v)(s)\right] d s\right\| \\
& \leq \int_{0}^{t_{2}}\left\|\left[R\left(t_{1}-s\right)-R\left(t_{2}-s\right)\right]\left[f\left(s, v_{s}\right)+k(v)(s)\right]\right\| d s+\int_{t_{2}}^{t_{1}} \|\left[R ( t _ { 1 } - s ) \| \| \left[f\left(s, v_{s}\right)+k(v)(s) \| d s\right.\right. \\
& \leq q\|v\|_{[-r, T]}\left[\int_{0}^{t_{2}} \int_{0}^{\infty} \sigma\left\|\left[\left(t_{1}-s\right)^{q-1}-\left(t_{2}-s\right)^{q-1}\right] \xi_{q}(\sigma) S\left(\left(t_{1}-s\right)^{q} \sigma\right)\right\|\left(\mu(s)+m^{*}\right) d \sigma d s\right. \\
& \left.\quad+\int_{0}^{t_{2}} \int_{0}^{\infty} \sigma\left(t_{2}-s\right)^{q-1} \xi_{q}(\sigma)\left\|S\left(\left(t_{1}-s\right)^{q} \sigma\right)-S\left(\left(t_{2}-s\right)^{q} \sigma\right)\right\|\left(\mu(s)+m^{*}\right) d \sigma d s\right] \\
& \quad+\frac{q M\|v\|_{[-r, T]}}{\Gamma(1+q)} \int_{t_{2}}^{t_{1}}\left(t_{1}-s\right)^{q-1}\left(\mu(s)+m^{*}\right) d s .
\end{aligned}
$$

Clearly, the first term on the right-hand side of (3.17) tends to 0 as $t_{2} \rightarrow t_{1}$. The second term on the right-hand side of (3.17) tends to 0 as $t_{2} \rightarrow t_{1}$ as a consequence of the continuity of $S(t)$ in the uniform operator topology for $t>0$. It is easy to see that the third term on the right-hand side of (3.17) tends to 0 as $t_{2} \rightarrow t_{1}$.

Thus, the set $\left\{\left(\mathcal{F}_{2} v\right)(\cdot): v \in \Omega\right\}$ is equicontinuous, then $\bmod _{c}\left(\mathcal{F}_{2} \Omega\right)=0$.

Since

$$
\bmod _{c}(\mathcal{F} \Omega) \leq \sum_{i=1}^{2} \bmod _{c}\left(\mathcal{F}_{i} \Omega\right) \leq M_{0} L_{h} \bmod _{c}(\Omega)
$$

then $\bmod _{c}(\Omega)=0$, which yields from (3.7). Hence

$$
\beta(\Omega)=(0,0) \text {. }
$$

The regularity property of $\beta$ implies the relative compactness of $\Omega$.

Now, it follows from Definition 2.7 that $\mathcal{F}$ is $\beta$-condensing.

Choose

$$
\rho>\frac{\tilde{N}+M\left(N+\|\phi(0)\|+M_{0} M_{1}(\tilde{N}+1)\right)+M_{0} M_{1}+\frac{C_{1-\alpha} \Gamma(1+\alpha)}{\alpha \Gamma(1+q \alpha)} M_{1} T^{q \alpha}}{1-M_{0} M_{1}},
$$

where $\tilde{N}:=N+\|\phi\|_{[-r, 0]}$.

Consider the set

$$
B_{\rho}=\left\{v \in C([-r, T], X):\|v\|_{[-r, T]} \leq \rho\right\} .
$$


Let us introduce in the space $C([-r, T], X)$ the equivalent norm defined as

$$
\|v\|_{\mathcal{c}}=\sup _{t \in[-r, 0]}\|v(t)\|+\sup _{t \in[0, T]}\left(e^{-L t} \sup _{s \in[0, t]}\|v(s)\|\right) .
$$

Noting

$$
\begin{aligned}
\left\|(-A)^{\alpha} h\left(t, v_{t}\right)\right\| & \leq M_{1}\left(\|v\|_{[-r, 0]}+\sup _{s \in[0, t]}\|v(s)\|+1\right) \\
& \leq M_{1} e^{L t}\left(\|v\|_{[-r, 0]}+e^{-L t} \sup _{s \in[0, t]}\|v(s)\|\right)+M_{1} \\
& \leq M_{1}\left(e^{L t}\|v\|_{c}+1\right)
\end{aligned}
$$

and

$$
\begin{aligned}
\left\|h\left(t, v_{t}\right)\right\| & =\left\|(-A)^{-\alpha}(-A)^{\alpha} h\left(t, v_{t}\right)\right\| \\
& \leq M_{0} M_{1}\left(e^{L t}\|v\|_{c}+1\right),
\end{aligned}
$$

for $t \in[0, T]$, wehave

$$
\begin{gathered}
\left\|Q(t)(g(v)(0)+\phi(0)-h(0, \phi+g(v)))+h\left(t, v_{t}\right)\right\| \\
\leq M\left(N+\|\phi(0)\|+M_{0} M_{1}(\tilde{N}+1)\right)+M_{0} M_{1}\left(e^{L t}\|v\|_{c}+1\right),
\end{gathered}
$$

then

$$
\begin{aligned}
& \left\|\mathcal{F}_{1} v\right\|_{c} \\
\leq & \tilde{N}+\sup _{t \in[0, T]}\left(e^{-L t} \sup _{s \in[0, t]}\left\|Q(s)(g(v)(0)+\phi(0)-h(0, \phi+g(v)))+h\left(s, v_{s}\right)\right\|\right) \\
\leq & \tilde{N}+M\left(N+\|\phi(0)\|+M_{0} M_{1}(\tilde{N}+1)\right)+M_{0} M_{1}\left(\|v\|_{c}+1\right) .
\end{aligned}
$$

Moreover, for $t \in[0, T]$, from (2.10) and (3.18) we have

$$
\begin{aligned}
& \left\|\int_{0}^{t} A R(t-s) h\left(s, v_{s}\right) d s\right\| \\
\leq & \int_{0}^{t}\left\|(-A)^{1-\alpha} R(t-s)(-A)^{\alpha} h\left(s, v_{s}\right)\right\| d s \\
\leq & \frac{q C_{1-\alpha} \Gamma(1+\alpha)}{\Gamma(1+q \alpha)} M_{1} \int_{0}^{t}(t-s)^{q \alpha-1}\left(e^{L s}\|v\|_{c}+1\right) d s,
\end{aligned}
$$

and from (2.9), (Hf)(1) and (Ha)(1) we get

$$
\begin{aligned}
& \left\|\int_{0}^{t} R(t-s)\left[f\left(s, v_{s}\right)+k(v)(s)\right] d s\right\| \\
\leq & \frac{q M}{\Gamma(1+q)} \int_{0}^{t}(t-s)^{q-1}\left(\mu(s)+m^{*}\right)\left(\|v\|_{[-r, 0]}+\sup _{\tau \in[0, s]}\|v(\tau)\|\right) d s \\
\leq & \frac{q M}{\Gamma(1+q)}\left[\int_{0}^{t}(t-s)^{q-1}\left(\mu(s)+m^{*}\right)\left(e^{L s}\|v\|_{C}\right) d s\right] .
\end{aligned}
$$


Therefore, from (3.19) and (3.20), we obtain

$$
\begin{aligned}
\left\|\mathcal{F}_{2} v\right\|_{C}= & \sup _{t \in[0, T]}\left(e^{-L t} \sup _{s \in[0, t]}\left\|\int_{0}^{s} A R(s-\tau) h\left(\tau, v_{\tau}\right) d \tau+\int_{0}^{s} R(s-\tau)\left[f\left(\tau, v_{\tau}\right)+k(v)(\tau)\right] d \tau\right\|\right) \\
\leq & \frac{q C_{1-\alpha} \Gamma(1+\alpha)}{\Gamma(1+q \alpha)} M_{1} \sup _{t \in[0, T]} \int_{0}^{t}(t-s)^{q \alpha-1}\left(e^{-L(t-s)}\|v\|_{\mathcal{C}}+e^{-L t}\right) d s \\
& +\frac{q M}{\Gamma(1+q)} \sup _{t \in[0, T]} \int_{0}^{t}(t-s)^{q-1}\left(\mu(s)+m^{*}\right)\left(e^{-L(t-s)}\|v\|_{C}\right) d s \\
\leq & \frac{q C_{1-\alpha} \Gamma(1+\alpha)}{\Gamma(1+q \alpha)} M_{1}\|v\|_{C} \sup _{t \in[0, T]} \int_{0}^{t}(t-s)^{q \alpha-1} e^{-L(t-s)} d s+\frac{C_{1-\alpha} \Gamma(1+\alpha)}{\alpha \Gamma(1+q \alpha)} M_{1} T^{q \alpha} \\
& +\frac{q M}{\Gamma(1+q)}\|v\|_{C} \sup _{t \in[0, T]} \int_{0}^{t}(t-s)^{q-1}\left(\mu(s)+m^{*}\right) e^{-L(t-s)} d s .
\end{aligned}
$$

Let $L \rightarrow+\infty$, we obtain

$$
\left\|\mathcal{F}_{2} v\right\|_{C} \leq \frac{C_{1-\alpha} \Gamma(1+\alpha)}{\alpha \Gamma(1+q \alpha)} M_{1} T^{q \alpha}
$$

Hence

$$
\begin{aligned}
\|\mathcal{F} v\|_{\mathcal{C}} \leq & \left\|\mathcal{F}_{1} v\right\|_{\mathcal{C}}+\left\|\mathcal{F}_{2} v\right\|_{\mathcal{C}} \\
\leq & \widetilde{N}+M\left(N+\|\phi(0)\|+M_{0} M_{1}(\widetilde{N}+1)\right)+M_{0} M_{1}\left(\|v\|_{\mathcal{C}}+1\right) \\
& +\frac{C_{1-\alpha} \Gamma(1+\alpha)}{\alpha \Gamma(1+q \alpha)} M_{1} T^{q \alpha} \\
\leq &
\end{aligned}
$$

Now, we show that there exists some $\rho>0$ such that $\mathcal{F} B_{\rho} \subset B_{\rho}$. According to Theorem 2.8, problem (1.1) has at least one mild solution.

Next, for $\widehat{\delta} \in(0,1]$, we consider the following one-parameter family of maps

$$
\begin{aligned}
& \Pi:[0,1] \times C([-r, T], X) \rightarrow C([-r, T], X) \\
& (\widehat{\delta}, v) \rightarrow \Pi(\widehat{\delta}, v)=\widehat{\delta} \mathcal{F}(v) .
\end{aligned}
$$

We will prove that the fixed point set of the family $\Pi$,

$$
\operatorname{Fix} \Pi=\{v \in \Pi(\widehat{\delta}, v) \text { for some } \widehat{\delta} \in(0,1]\}
$$

is a priori bounded.

Noting that the Hölder inequality, we have

$$
\int_{0}^{t}(t-s)^{q \alpha-1} \mu(s) d s \leq l_{p, q} \cdot t^{\frac{p q \alpha-1}{p}} \cdot\|\mu\|_{L^{p}} \leq l_{p, q} \cdot T^{q \alpha-\frac{1}{p}} \cdot\|\mu\|_{L^{p}} .
$$


Let $v \in$ Fix $\Pi$, for $t \in[0, T]$, we have

$$
\begin{aligned}
\|v(t)\| \leq & \|Q(t)(g(v)(0)+\phi(0)-h(0, \phi+g(v)))\|+\left\|h\left(t, v_{t}\right)\right\|+\int_{0}^{t}\left\|A R(t-s) h\left(s, v_{s}\right)\right\| d s \\
& +\int_{0}^{t}\left\|R(t-s)\left[f\left(s, v_{s}\right)+k(v)(s)\right]\right\| d s \\
\leq & M(N+\|\phi(0)\|)+M M_{0} M_{1}(\tilde{N}+1)+M_{0} M_{1}\left(\left\|v_{t}\right\|_{[-r, 0]}+1\right) \\
& +\frac{q M_{1} C_{1-\alpha} \Gamma(1+\alpha)}{\Gamma(1+q \alpha)} \int_{0}^{t}(t-s)^{q \alpha-1}\left(\left\|v_{s}\right\|_{[-r, 0]}+1\right) d s \\
& +\frac{q M}{\Gamma(1+q)} \int_{0}^{t}(t-s)^{q-1}\left(\mu(s)\left\|v_{s}\right\|_{[-r, 0]}+\int_{0}^{s} m(s, \tau)\left\|v_{\tau}\right\|_{[-r, 0]} d \tau\right) d s \\
\leq & M(N+\|\phi(0)\|)+M M_{0} M_{1}(\tilde{N}+1)+M_{0} M_{1}\left(\tilde{N}+\sup _{s \in[0, t]}\|v(s)\|+1\right) \\
& +\frac{q M_{1} C_{1-\alpha} \Gamma(1+\alpha)}{\Gamma(1+q \alpha)} \int_{0}^{t}(t-s)^{q \alpha-1}\left(\tilde{N}+\sup _{\tau \in[0, s]}\|v(\tau)\|+1\right) d s \\
& +\frac{q M T^{q(1-\alpha)}}{\Gamma(1+q)} \int_{0}^{t}(t-s)^{q \alpha-1}\left(\mu(s)+m^{*}\right)\left(\tilde{N}+\sup _{\tau \in[0, s]}\|v(\tau)\|\right) d s \\
\leq & a_{0}+a_{1} \sup _{s \in[0, t]}\|v(s)\|+a_{2} \int_{0}^{t}(t-s)^{q \alpha-1} \sup _{\tau \in[0, s]}\|v(\tau)\| d s,
\end{aligned}
$$

where

$$
\begin{aligned}
a_{0}= & M\left(N+\|\phi(0)\|+(M+1) M_{0} M_{1}(\tilde{N}+1)+\frac{M_{1} C_{1-\alpha} \Gamma(1+\alpha)}{\alpha \Gamma(1+q \alpha)} T^{q \alpha}(\tilde{N}+1)\right. \\
& +\frac{q M}{\Gamma(1+q)} l_{p, q} T{ }^{q-\frac{1}{p}}\|\mu\|_{L^{p}} \tilde{N}+\frac{M m^{*} T^{q}}{\alpha \Gamma(1+q)} \tilde{N}, \\
a_{1}= & M_{0} M_{1}+\frac{q M}{\Gamma(1+q)} l_{p, q} T-\frac{1}{p}\|\mu\|_{L^{p}}, \\
a_{2}= & \frac{q M_{1} C_{1-\alpha} \Gamma(1+\alpha)}{\Gamma(1+q \alpha)}+\frac{q M m^{*} T^{q(1-\alpha)}}{\Gamma(1+q)} .
\end{aligned}
$$

We denote $\bar{\gamma}(t):=\sup _{s \in[0, t]}\|v(s)\|$. Let $\tilde{t} \in[0, t]$ such that $\bar{\gamma}(t)=\|v(\tilde{t})\|$. Then, by (3.22), we can see

$$
\bar{\gamma}(t) \leq a_{0}+a_{1} \bar{\gamma}(t)+a_{2} \int_{0}^{t}(t-s)^{q \alpha-1} \bar{\gamma}(s) d s .
$$

By Lemma 2.10, there exists a constant $\kappa=\kappa(q \alpha)$ such that

$$
\bar{\gamma}(t) \leq \frac{a_{0}}{1-a_{1}}+\frac{k a_{0} a_{2}}{\left(1-a_{1}\right)^{2}} \int_{0}^{t}(t-s)^{q \alpha-1} d s \leq \frac{a_{0}}{1-a_{1}}+\frac{k a_{0} a_{2} T^{q \alpha}}{q \alpha\left(1-a_{1}\right)^{2}}:=\tilde{\varpi} .
$$

Hence $\sup _{t \in[0, T]}\|v(t)\| \leq \tilde{\varpi}$. 
Consequently

$$
\|v\|_{[-r, T]} \leq\|v\|_{[-r, 0]}+\|v\|_{[0, T]} \leq \tilde{N}+\tilde{\varpi} .
$$

Now we consider a closed ball

$$
B_{R}=\left\{v \in C([-r, T], X):\|v\|_{[-r, T]} \leq R\right\} \subset C([-r, T], X) .
$$

We take the radius $R>0$ large enough to contain the set Fix $\Pi$ inside itself. Moreover, from the proof above, $\mathcal{F}: B_{R} \rightarrow C([-r, T], X)$ is $\beta$-condensing and it remains to apply Theorem 2.9. $\square$

If we assume $\mu(\cdot)\left\lfloor C\left([0, T], \mathbf{R}^{+}\right)\right.$in $(\mathrm{Hf})(1)$, then we have

Theorem 3.2. Assume that (Hf), (Hh), ( $\mathrm{Ha})$ and $(\mathrm{Hg})$ are satisfied, if

(1) $M_{0} \cdot \max \left\{M_{1}, L_{h}\right\}<1$,

(2) $L_{g}^{*}\left(1+M M_{0} M_{2}+M\right)+M_{0} M_{2}<1$,

where $L_{g}^{*}=\sup _{t \in[-r, 0]} L_{g}(t)$. Then the mild solutions set of problem (1.1) is a nonempty compact subset of the space $C([-r, T], X)$.

From the proof in Theorem 3.1, we can see that Theorem 3.2 holds.

\section{Application}

In this section, let $X=L^{2}([0, \pi])$, we consider the following integrodifferential problem:

$$
\left\{\begin{array}{l}
\frac{\partial^{q}}{\partial t^{q}}\left[u(t, x)-e^{-t} \int_{0}^{\pi} \frac{\gamma_{1}(x, y) u_{t}(\theta, y)}{1+\left|u_{t}(\theta, y)\right|} d y\right]=\frac{\partial^{2}}{\partial x^{2}} u(t, x) \\
\quad+\sqrt[k]{t} \cdot \sin \left(\left|u_{t}(\theta, x)\right|\right)+\int_{0}^{t}(t-s)^{-\frac{2}{3}} \int_{-r}^{0} \gamma_{2}(\theta) \cdot \sin \left(\frac{\left|u_{s}(\theta, x)\right|}{s^{\frac{1}{3}}}\right) d \theta d s, \\
u(t, 0)=u(t, \pi)=0, \\
u(\theta, x)=\int_{0}^{\pi} c(x, y) \sin (1+|u(\theta, y)|) d y+u_{0}(\theta, x), \quad-r \leq \theta \leq 0
\end{array}\right.
$$

where $t \in[0,1], r>0, x \in[0, \pi]$ and $u_{t}(\theta, x)=u(t+\theta, x) \cdot \gamma_{1}:[0, \pi] \times[0, \pi] \rightarrow \mathbf{R}, \gamma_{2}$ $:[-r, 0] \rightarrow \mathbf{R}$ and $c(x, y) \in L^{2}([0, \pi] \times[0, \pi], \mathbf{R})$ are functions to be specified later.

To treat the above problem, we define

$$
\begin{aligned}
D(A) & =H^{2}([0, \pi]) \cap H_{0}{ }^{1}([0, \pi]), \\
A u & =-u^{\prime \prime} .
\end{aligned}
$$

The operator $-A$ is the infinitesimal generator of an analytic semigroup $\{T(t)\}_{t \geq 0}$ on $X$. Moreover, $A$ has a discrete spectrum, the eigenvalues are $n^{2}, n \in N$, with the corresponding normalized eigenvectors $\omega_{n}(x)=\sqrt{\frac{2}{\pi}} \sin (n x)$ and the following properties are satisfied:

(a) if $w \in D(A)$, then $A \omega=\sum_{n=1}^{\infty} n^{2}\left\langle\omega, \omega_{n}\right\rangle \omega_{n}$.

(b) For each $\omega \in X, T(t) \omega=\sum_{n=1}^{\infty} \exp \left(-n^{2} t\right)\left\langle\omega, \omega_{n}\right\rangle \omega_{n}$ and $\|T(t)\| \leq 1$ for $t \geq 0$.

(c) For each $\omega \in X, A^{-\frac{1}{2}} \omega=\sum_{n=1}^{\infty} \frac{1}{n}\left\langle\omega, \omega_{n}\right\rangle \omega_{n}$. In particular, $\left\|A^{-\frac{1}{2}}\right\|=1$. 
(d) $A^{\frac{1}{2}}$ is given by $A^{\frac{1}{2}} \omega=\sum_{n=1}^{\infty} n\left\langle\omega, \omega_{n}\right\rangle \omega_{n}$ with domain

$$
D\left(A^{\frac{1}{2}}\right)=\left\{\omega \in X: \sum_{n=1}^{\infty} n\left\langle\omega, \omega_{n}\right\rangle \omega_{n} \in X\right\} .
$$

We assume that the following conditions hold.

(1) The function $\gamma_{1}:[0, \pi] \times[0, \pi] \rightarrow \mathbf{R}$ is continuously differential with $\gamma_{1}(0, y)=\gamma_{1}$ $(\pi, y)=0$ for $y \in[0, \pi]$ and

$$
\left[\int_{0}^{\pi} \int_{0}^{\pi}\left(\frac{\partial \gamma_{1}(x, y)}{\partial x}\right)^{2} d y d x\right]^{\frac{1}{2}}<\infty
$$

(2) The function $\gamma_{2}:[-r, 0] \rightarrow \mathbf{R}$ is a continuous function, and $\int_{-r}^{0}\left|\gamma_{2}(\theta)\right| d \theta<\infty$.

(3) The function $c(x, y), x, y \in[0, \pi]$, is measurable and there exists a constant $N$ such that

$$
\int_{0}^{\pi}\|c(x, y)\| d y \leq N
$$

For $x \in[0, \pi]$ and $\phi \in C([-r, 0], X)$, we set

$$
\begin{aligned}
v(t)(x) & =u(t, x) \\
\phi(\theta)(x) & =u_{0}(\theta, x), \theta \in[-r, 0] \\
h(t, \varphi)(x) & =e^{-t} \int_{0}^{\pi} \frac{\gamma_{1}(x, y) \varphi(\theta)(y)}{1+|\varphi(\theta)(y)|} d y \\
g(\varphi(\theta))(x) & =\int_{0}^{\pi} c(x, y) \sin (1+|\varphi(\theta)(\gamma)|) d y \\
f(t, \varphi)(x) & =\sqrt[k]{t} \cdot \sin (|\varphi(\theta)(x)|), \\
a(t, s, \varphi)(x) & =(t-s)-\frac{2}{3} \int_{-r}^{0} \gamma_{2}(\theta) \cdot \sin \left(\frac{|\varphi(\theta)(x)|}{s^{\frac{1}{3}}}\right) d \theta
\end{aligned}
$$

where $\sqrt[k]{t} \in L^{P}\left([0,1], \mathbf{R}^{+}\right)\left(p>\frac{2}{q}\right)$.

Then the above Equation (4.1) can be reformulated as the abstract (1.1).

For $t \in[0,1]$, we can see

$$
\|f(t, \varphi)\| \leq \sqrt[k]{t} \cdot\|\varphi\|_{[-r, 0]} .
$$

For any $\varphi, \tilde{\varphi} \in C([-r, 0], X)$,

$$
\|f(t, \varphi)(x)-f(t, \tilde{\varphi})(x)\| \leq \sqrt[k]{t} \cdot\|\varphi(\theta)(x)-\tilde{\varphi}(\theta)(x)\| .
$$


Therefore, for any bounded set $D \subset C([-r, 0], X)$, we have

$$
X(f(t, D)) \leq \sqrt[k]{t} \cdot \sup _{-r \leq \theta \leq 0} \chi(D(\theta)), \quad t \in[0,1] .
$$

Noting that

$$
\begin{aligned}
\left\langle h(t, \varphi), \omega_{n}\right\rangle & =\int_{0}^{\pi} \omega_{n}(x)\left(e^{-t} \int_{0}^{\pi} \frac{\gamma_{1}(x, y) \varphi(\theta)(y)}{1+|\varphi(\theta)(y)|} d y\right) d x \\
& =\frac{e^{-t}}{n}\left\langle\int_{0}^{\pi} \frac{\partial}{\partial x}\left(\frac{\gamma_{1}(x, y) \varphi(\theta)(y)}{1+|\varphi(\theta)(y)|}\right) d y, \sqrt{\frac{2}{\pi}} \cos (n x)\right\rangle
\end{aligned}
$$

and

$$
\begin{aligned}
\left\|A^{\frac{1}{2}} h(t, \varphi)\right\| & =\left\|\sum_{n=1}^{\infty} n\left\langle h(t, \varphi), \omega_{n}\right\rangle \omega_{n}\right\| \\
& \leq\left[\int_{0}^{\pi} \int_{0}^{\pi}\left(\frac{\partial \gamma_{1}(x, y)}{\partial x}\right)^{2} d y d x\right]^{\frac{1}{2}}\left[\int_{0}^{\pi}(\varphi(\theta)(y))^{2} d y\right]^{\frac{1}{2}} \\
& \leq M_{1}\|\varphi\|_{[-r, 0]}
\end{aligned}
$$

and

$$
\begin{aligned}
& \left\|A^{\frac{1}{2}} h\left(t_{1}, \varphi\right)-A^{\frac{1}{2}} h\left(t_{2}, \tilde{\varphi}\right)\right\| \\
= & \left\|\sum_{n=1}^{\infty} n\left\langle h\left(t_{1}, \varphi\right)-h\left(t_{2}, \tilde{\varphi}\right), \omega_{n}\right\rangle \omega_{n}\right\| \\
\leq & \sqrt{\pi}\left[\int_{0}^{\pi} \int_{0}^{\pi}\left(\frac{\partial \gamma_{1}(x, y)}{\partial x}\right)^{2} d y d x\right]^{\frac{1}{2}}\left|t_{1}-t_{2}\right| \\
& +\left[\int_{0}^{\pi} \int_{0}^{\pi}\left(\frac{\partial \gamma_{1}(x, y)}{\partial x}\right)^{2} d y d x\right]^{\frac{1}{2}}\left[\int_{0}^{\pi}(\varphi(\theta)(y)-\tilde{\varphi}(\theta)(y))^{2} d y\right]^{\frac{1}{2}} \\
\leq & L_{h}\left(\left|t_{1}-t_{2}\right|+\|\varphi-\tilde{\varphi}\|_{[-r, 0]}\right),
\end{aligned}
$$

where $M_{1}=\left[\int_{0}^{\pi} \int_{0}^{\pi}\left(\frac{\partial \gamma_{1}(x, y)}{\partial x}\right)^{2} d y d x\right]^{\frac{1}{2}}, L_{h}=\sqrt{\pi}\left[\int_{0}^{\pi} \int_{0}^{\pi}\left(\frac{\partial \gamma_{1}(x, y)}{\partial x}\right)^{2} d y d x\right]^{\frac{1}{2}}$, we

can see that for any bounded set $D \subset C([-r, 0], X)$ and $t \in[0,1]$,

$$
\chi\left(A^{\frac{1}{2}} h(t, D)\right) \leq L_{h} \sup _{\theta \in[-r, 0]} \chi(D(\theta)) .
$$


Moreover,

$$
\begin{aligned}
\|a(t, s, \varphi)(x)\| & =\left\|(t-s)^{-\frac{2}{3}} \int_{-r}^{0} \gamma_{2}(\theta) \cdot \sin \left(\frac{|\varphi(\theta)(x)|}{s^{\frac{1}{3}}}\right) d \theta\right\| \\
& \leq(t-s)^{-\frac{2}{3}} s^{-\frac{1}{3}} \int_{-r}^{0}\left|\gamma_{2}(\theta)\right| d \theta \cdot\|\varphi\|_{[-r, 0],}
\end{aligned}
$$

where $m(t, s):=(t-s)^{-\frac{2}{3}} s^{-\frac{1}{3}} \int_{-r}^{0}\left|\gamma_{2}(\theta)\right| d \theta$. Then, we obtain

$$
\sup _{t \in[0,1]} \int_{0}^{t} m(t, s) d s=\int_{-r}^{0}\left|\gamma_{2}(\theta)\right| d \theta \cdot \sup _{t \in[0,1]} \int_{0}^{t}(t-s)^{-\frac{2}{3}} s^{-\frac{1}{3}} d s=B\left(\frac{2}{3}, \frac{1}{3}\right) \cdot \int_{-r}^{0}\left|\gamma_{2}(\theta)\right| d \theta
$$

and

$$
\|a(t, s, \varphi)(x)-a(t, s, \tilde{\varphi})(x)\| \leq(t-s)^{-\frac{2}{3}} s^{-\frac{1}{3}} \int_{-r}^{0}\left|\gamma_{2}(\theta)\right|\|\varphi(\theta)(x)-\tilde{\varphi}(\theta)(x)\| d \theta .
$$

Hence, for any bounded set $D \subset C([-r, 0], X)$, we have

$$
\chi(a(t, s, D)) \leq \zeta(t, s) \sup _{-r \leq \theta \leq 0} \chi(D(\theta)),
$$

where

$$
\zeta(t, s):=(t-s)^{-\frac{2}{3}} s^{-\frac{1}{3}} \int_{-r}^{0}\left|\gamma_{2}(\theta)\right| d \theta
$$

$\sup _{t \in[0,1]} \int_{0}^{t} \zeta(t, s) d s=B\left(\frac{2}{3}, \frac{1}{3}\right) \cdot \int_{-r}^{0}\left|\gamma_{2}(\theta)\right| d \theta$.

For $\varphi, \tilde{\varphi} \in C([-r, 0], X), \theta \in[-r, 0]$, we can get

$$
\|g(\varphi)(x)-g(\tilde{\varphi})(x)\| \leq\left(\int_{0}^{\pi} \int_{0}^{\pi} c^{2}(x, y) d y d x\right)^{1 / 2} \cdot\|\varphi-\tilde{\varphi}\|:=\tilde{L}_{g} \cdot\|\varphi-\tilde{\varphi}\| .
$$

Moreover,

$$
\|g(\varphi)(x)\| \leq \int_{0}^{\pi}\|c(x, y)\| d y \leq N
$$

Suppose further that there exist constants $\tilde{M}^{*}, \tilde{M}^{\prime} \in(0,1)$ such that

(1) $L_{h}+\frac{1}{\Gamma(q)}\left(\frac{2 p-2}{p q-2}\right)^{\frac{p-1}{p}}\left(\frac{k}{k+p}\right)^{\frac{1}{p}}<\tilde{M}^{*}$;

(2) $\tilde{L}_{g}\left(2+L_{h}\right)+L_{h}<\tilde{M}^{\prime}$,

then (4.1) has a mild solution by Theorem 3.1.

\section{Acknowledgements}

This work was supported by the NSF of Yunnan Province (2009ZC054M).

\section{Competing interests}

The authors declare that they have no competing interests. 


\section{References}

1. El-Borai, MM: Some probability densities and fundamental solutions of fractional evolution equations. Chaos Solitons Fract. 149, 823-831 (2004)

2. Li, F: Solvability of nonautonomous fractional integrodifferential equations with infinite delay. Adv Diff Equ 2011, 18 (2011). Article ID 806729. doi:10.1186/1687-1847-2011-18

3. Li, F: An existence result for fractional differential equations of neutral type with infinite delay. Electron J Qual Theory Diff Equ. 2011(52), 1-15 (2011)

4. Mainardi, F, Paradisi, P, Gorenflo, R, Kertesz, J, Kondor, I: Probability distributions generated by fractional diffusion equations. Econophysics: An Emerging Science. Kluwer, Dordrecht (2000)

5. Mophou, GM, N'Guérékata, GM: Existence of mild solution for some fractional differential equations with nonlocal conditions. Semigroup Forum. 79(2), 315-322 (2009). doi:10.1007/s00233-008-9117-x

6. Mophou, GM, N'Guérékata, GM: Existence of mild solutions for some semilinear neutral fractional functional evolution equations with infinite delay. Appl Math Comput. 216, 61-69 (2010). doi:10.1016/j.amc.2009.12.062

7. Mophou, GM, N'Guérékata, GM: A note on a semilinear fractional differential equation of neutral type with infinite delay. Adv Diff Equ 2010, 8 (2010). Article ID 674630

8. N'Guérékata, GM: A Cauchy problem for some fractional abstract differential equation with non local conditions. Nonlinear Anal Theory Methods Appl. 70(5), 1873-1876 (2009). doi:10.1016/j.na.2008.02.087

9. Podlubny, I: Fractional Differential Equations. Academic Press, San Diego (1999)

10. Ren, Y, Qin, Y, Sakthivel, R: Existence results for fractional order semilinear integro-differential evolution equations with infinite delay. Integr Equ Operat Theory. 67, 33-49 (2010). doi:10.1007/s00020-010-1767-x

11. Liang, J, Xiao, TJ: Solvability of the Cauchy problem for infinite delay equations. Nonlinear Anal Theory Methods Appl. 58, 271-297 (2004). doi:10.1016/j.na.2004.05.005

12. Xiao, TJ, Liang, J: Blow-up and global existence of solutions to integral equations with infinite delay in Banach spaces. Nonlinear Anal Theory Methods Appl. 71, e1442-e1447 (2009). doi:10.1016/j.na.2009.01.204

13. Liang, J, Liu, JH, Xiao, TJ: Nonlocal Cauchy problems governed by compact operator families. Nonlinear Anal Theory Methods Appl. 57(2), 183-189 (2004). doi:10.1016/j.na.2004.02.007

14. Liang, J, Xiao, TJ: Semilinear integrodifferential equations with nonlocal initial conditions. Comput Math Appl. 47(6-7), 863-875 (2004). doi:10.1016/S0898-1221(04)90071-5

15. Xiao, TJ, Liang, J: Existence of classical solutions to nonautonomous nonlocal parabolic problems. Nonlinear Anal Theory Methods Appl. 63, e225-e232 (2005). doi:10.1016/j.na.2005.02.067

16. Pazy, A: Semigroups of Linear Operators and Applications to Partial Differential Equations. In Appl Math Sci, vol. 44, Springer Verlag, New York (1983)

17. Akhmerov, RR, Kamenskii, MI, Potapov, AS, Rodkina, AE, Sadovskii, BN: Measures of Noncompactness and Condensing Operators. Birkhäauser, Boston/Basel/Berlin (1992)

18. Kamenskii, M, Obukhovskii, V, Zecca, P: Condensing Multivalued Maps and Semi-linear Differential Inclusions in Banach Spaces. In de Gruyter Ser Nonlinear Anal Appl, vol. 7,Walter de Gruyter, Berlin/New York (2001)

19. Henry, D: Geometric Theory of Semilinear Parabolic Partial Differential Equations. Springer, Berlin, Germany (1989)

doi:10.1186/1687-1847-2012-47

Cite this article as: Li: Nonlocal cauchy problem for delay fractional integrodifferential equations of neutral type. Advances in Difference Equations 2012 2012:47.

\section{Submit your manuscript to a SpringerOpen ${ }^{\circ}$ journal and benefit from:}

Convenient online submission

- Rigorous peer review

- Immediate publication on acceptance

- Open access: articles freely available online

- High visibility within the field

- Retaining the copyright to your article

Submit your next manuscript at $\mathbf{s p r i n g e r o p e n . c o m ~}$ 